\title{
Switching from Abacavir/Lamivudine to Tenofovir DF/Emtricitabine Reduces Biomarkers of Inflammation: A Randomized Proof of Concept Study
}

Alozie $\mathrm{O}^{1 *}$, Prosser $\mathrm{R}^{2,3}$, Huppler Hullsiek $\mathrm{K}^{3}$, Duprez $\mathrm{D}^{3}$, Rhame $\mathrm{F}^{3,4}$, Henry $\mathrm{WK}^{2,3}$ and Baker $\mathrm{JV}^{2,3}$

${ }^{1}$ Texas Tech University Health Sciences Center (TTUHSC), EI Paso, TX, USA

${ }^{2}$ HIV Program, Hennepin County Medical Center (HCMC), Minneapolis, MN, USA

${ }^{3}$ University of Minnesota, Minneapolis, MN, USA

${ }^{4}$ Abbott Northwestern Hospitals and Clinics, Minneapolis, MN, USA

\begin{abstract}
Background:_Abacavir use has been associated with cardiovascular disease (CVD) risk, but this effect has not been consistent across studies.

Methods: To explore abacavir-related CVD risk we studied 27 HIV-positive participants taking fixed-dose abacavir/ lamivudine-based antiretroviral therapy (ART) with viral suppression and randomized them to remain on their current regimen $(n=13)$ or switch the nucleoside component to tenofovir disoproxil fumarate (DF)/emtricitabine $(n=14)$. Plasma biomarkers were measured at baseline and at 1 and 6 months.

Results: At baseline, median (IQR) age was 46 years (41-53) and CD4+ count 620 cells $/ \mathrm{mm}^{3}$ (477-836). There were no baseline differences in individual CVD risk factors between groups, however, 10-year Framingham Risk Score (FRS) trended higher for those taking abacavir (8.5\%) versus tenofovir DF (4.7\%). Switching to a tenofovir DF-based ART regimen, compared with staying on abacavir-based ART, was associated with a $79 \%$ lower level of high sensitivity C-reactive protein (hsCRP; $p=0.04$ ) and a $52 \%$ lower inflammatory/coagulation rank composite (consisting of hsCRP, interleukin- 6 and D-dimer levels). These findings were not attenuated after adjusting for 10 -year FRS (-79\% for hsCRP, $p=0.06 ;-50 \%$ for inflammatory composite, $p=0.003$ ).
\end{abstract}

Conclusion: Larger, prospective, randomized studies are needed to verify whether switching from abacavir/ lamivudine- to tenofovir DF/emtricitabine-based ART, in the context of viral suppression, reduces inflammation and corresponding CVD risk.

Keywords: HIV; CVD; Inflammation; ART

\section{Introduction}

The spectrum of morbsidity and mortality among HIV positive persons is now more commonly characterized by long-term complications such as atherosclerotic cardiovascular disease (CVD) [1]. As a result, HIV clinical management now emphasizes modifying traditional and HIV-specific risk factors that contribute to CVD risk. HIV factors, in part, include toxicities from individual components of antiretroviral therapy (ART) [2]. Exposure to the nucleoside reverse transcriptase inhibitor (NRTI) abacavir has been associated with increased CVD event risk in some studies, but not in others [3-10]. Few studies have prospectively explored the potential adverse effects of abacavir among virally suppressed patients, data are needed to determine whether tailoring individual components of ART (e.g., stopping abacavir) is a viable strategy to reduce CVD risk.

To explore potential CVD-related toxicity from abacavir exposure, we measured plasma biomarkers from stored specimens as part of a proof of concept pilot study assessing the effects of switching from an abacavir-to a tenofovir disoproxil fumarate (DF)-based ART regimen.

\section{Methods}

\section{Study design}

Participants were recruited from Hennepin County Medical Center (HCMC) and Abbott Northwestern (ANW) HIV Clinics in Minneapolis, Minnesota, USA.We enrolled medically stable HIVpositive participants with viral suppression (HIV RNA $<48$ copies/
$\mathrm{mL}$ ) for $\geq 3$ months, taking fixed-dose abacavir/lamivudine and one additional antiretroviral medication. Exclusion criteria included: a creatinine clearance $<50 \mathrm{~mL} / \mathrm{min}$ (estimated by Cockcroft-Gault formula), a contraindication to taking tenofovir $\mathrm{DF}$, and the presence of resistance mutations to abacavir, tenofovir DF, lamivudine or emtricitabine. The randomized intervention consisted of: a) maintaining their current regimen ('ABC group'), or b) switching to tenofovirDF/emtricitabine-based ART ('TDF group'), while maintaining the third component of ART in place. The study goal was to explore and quantify any treatment effect on vascular inflammation and endothelial dysfunction, with additional analysis of inflammatory biomarkers planned a priori, that might inform additional analyses of stored specimens from larger clinical trials [11]. Planned sample size for this pilot phase was based on ability to recruit. All participants underwent informed consent. The study protocol was approved by

*Corresponding author: Alozie O, Texas Tech University Health Sciences Center (TTUHSC), El Paso, TX, USA, Tel: 915996 1202; E-mail: ogechika.alozie@ttuhsc.edu

Received January 01, 2014; Accepted January 27, 2014; Published January 31 2014

Citation: Alozie O, Prosser R, Huppler Hullsiek K, Duprez D, Rhame F, et al. (2014 Switching from Abacavir/Lamivudine to Tenofovir DF/Emtricitabine Reduces Biomarkers of Inflammation: A Randomized Proof of Concept Study. J AIDS Clin Res 5: 278. doi:10.4172/2155-6113.1000278

Copyright: ( 2014 Alozie O, et al. This is an open-access article distributed unde the terms of the Creative Commons Attribution License, which permits unrestricted use, distribution, and reproduction in any medium, provided the original author and source are credited. 
ethics committees at both sites and registered at ClinicalTrials.gov (NCT\#00998582).

\section{Laboratory methods}

Biomarker levels, from cryo preserved plasma specimens collected at month 1 and 6 , were measured at the Laboratory for Clinical Biochemistry Research at the University of Vermont using stored plasma. Inflammation and coagulation was assessed via levels of high sensitivity C-reactive Protein (hsCRP) by $\mathrm{NB}^{\text {tw } I I}$ nephelometer $\mathrm{N}$ Antiserum to Human CRP (Siemens Diagnostics), interleukin-6 (IL-6) by Chemiluminescent Sandwich ELISA (R\&D Systems), and D-dimer by immunoturbidometric methods (Diagnostica Stago). Vascular inflammation and endothelial dysfunction was indirectly assessed via the following biomarkers: soluble intercellular adhesion moledule-1 (sICAM) with ELISA from Parameter Human Immunoassay (R\&D Systems), von Willebrand Factor (vWF) by Stago STA-R analyzer (Stago Diagnostics), serum thrombomodulin (sTM) by Chemiluminescent Sandwich ELISA (R\&D Systems), and asymmetric dimethylarginine (ADMA) by competitive enzyme-linked immunosorbent assay (Euroimmun).All samples were analyzed blinded to treatment group.

\section{Statistical methods}

Baseline medians were compared utilizing Wilcoxon tests; proportions were compared with chi-squared or Fisher's exact tests. Differences between randomized groups were estimated with generalized linear models adjusted for baseline biomarker level, with the primary comparison at month 6 . Biomarker levels were $\log _{\mathrm{e}}-$ transformed before analysis; results are reported as percent differences between treatment groups by exponentiating $\log _{\mathrm{e}}$-transformed values. Additional analyses adjusted for baseline 10-year Framingham Risk Score (FRS) for coronary heart disease event and estimated glomerular filtration rate (eGFR). A global assessment composite measure [12] was considered by grouping biomarkers together based on: inflammation/ coagulation (hsCRP, IL-6, and D-dimer) and vascular inflammation (sICAM, ADMA, vWF, sTM) assessments. We expected a similar treatment effect on the individual markers within each composite, so the intent in generating composite scores was to reduce measurement variability since the biomarkers within each panel represent overlapping biology. With the "rank-sum" method biomarker level values in each composite were ranked and then summed by treatment group. Models were used to compare the sum of the ranks between treatment groups. Two-sided p-values and 95\% confidence intervals (CIs) are cited, with no adjustments for multiple comparisons. Analyses were performed using SAS version 9.2 (SAS Institute) and R version 2.9.

\section{Results}

Twenty-seven participants were screened and randomized to either stay on abacavir/lamivudine- $(n=13)$ or switch to tenofovir DF/ emtricitabine- $(\mathrm{n}=14)$ based ART. Among randomized participants, 27 were seen at month one and 26 at month six visits. Baseline characteristics and biomarker levels are presented in Table 1. Individual CVD risk factors did not differ by randomized allocation, but the Framingham Risk Score (FRS) for 10-year risk of a coronary heart disease event tended to be higher among the abacavir (8.5\%) versus tenofovir $(4.7 \%)$ group $(\mathrm{p}=0.052)$. The third antiretroviral medication

\begin{tabular}{|c|c|c|c|c|}
\hline & \multirow[b]{2}{*}{ Overall } & \multicolumn{2}{|c|}{ Randomization Group } & \multirow[b]{2}{*}{$P$-value* } \\
\hline & & 'Abacavir group' & 'Tenofovir Group' & \\
\hline Total Sample, number & 27 & 13 & 14 & \\
\hline Age, median (IQR) years & $46[41,53]$ & $47[43,53]$ & $42.5[32,52]$ & 0.30 \\
\hline Male Gender, \# (\%) & $26(96.3 \%)$ & $12(92.3 \%)$ & $14(100.0 \%)$ & 0.30 \\
\hline Race/Ethnicity, \# (\%) & & & & 0.36 \\
\hline White & $22(81.5 \%)$ & $10(76.9 \%)$ & $12(85.7 \%)$ & \\
\hline African American & $4(14.8 \%)$ & $3(23.1 \%)$ & $1(7.1 \%)$ & \\
\hline Hispanic & $0(0.0 \%)$ & $0(0.0 \%)$ & $0(0.0 \%)$ & \\
\hline Other & $1(3.7 \%)$ & $0(0.0 \%)$ & $1(7.1 \%)$ & \\
\hline IDU, \# (\%) & & & & 0.13 \\
\hline Prior & $2(7)$ & $2(15)$ & $0(0)$ & \\
\hline Never & $25(93)$ & $11(85)$ & $14(100)$ & \\
\hline Hep B or C positive, \# (\%) & $3(11)$ & $2(15)$ & $1(7)$ & 0.30 \\
\hline Current Smoker, \# (\%) & $6(22)$ & $3(23)$ & $3(21)$ & 0.92 \\
\hline Blood pressure lowering therapy, \# (\%) & $4(16)$ & $4(31)$ & $0(0)$ & 0.10 \\
\hline Lipid lowering therapy, \# (\%) & $6(23)$ & $3(23)$ & $3(23)$ & $>0.99$ \\
\hline Tot. Chol., median (IQR) mg/dL & $201(178,238)$ & $205(185,214)$ & $189(178,243)$ & 0.75 \\
\hline LDL-C, median (IQR) mg/dL & $107(91,127)$ & $100(93,127)$ & $108(91,126)$ & 0.81 \\
\hline $\mathrm{HDL}-\mathrm{C}$, median (IQR) mg/dL & $43(36,55)$ & $42(36,48)$ & $49(36,59)$ & 0.27 \\
\hline Framingham Risk Score, median (IQR) \% coronary event over 10yrs & $6.0(3.6,10.4)$ & $8.5(5.6,12.8)$ & $4.7(2.3,7.9)$ & 0.052 \\
\hline \multicolumn{5}{|l|}{ Biomarkers } \\
\hline hsCRP, median (IQR) $\mu \mathrm{g} / \mathrm{mL}$ & $2.1(0.9,3.2)$ & $2.1(1.2,2.8)$ & $2.3(0.9,3.2)$ & 0.78 \\
\hline IL-6, median (IQR) pg/mL & $1.7(1.2,2.3)$ & $1.7(1.5,2.2)$ & $2.1(1.0,2.3)$ & 0.78 \\
\hline D-dimer, median (IQR) $\mu \mathrm{g} / \mathrm{mL}$ & $0.2(0.1,0.2)$ & $0.2(0.1,0.5)$ & $0.1(0.1,0.2)$ & 0.02 \\
\hline ADMA, median (IQR) $\mu \mathrm{mol} / \mathrm{L}$ & $0.6(0.5,0.7)$ & $0.6(0.5,0.7)$ & $0.6(0.5,0.8)$ & 0.47 \\
\hline sICAM-1, median (IQR) ng/mL & $228(184,274)$ & $228(172,274)$ & $227(190,253)$ & 0.98 \\
\hline vWF, median (IQR) \% & $122(108,153)$ & $133(110,183)$ & $120(108,133)$ & 0.34 \\
\hline sTM, median (IQR) pg/mL & $4944(4145,5945)$ & $5382(4096,5945)$ & $4882(4567,5561)$ & 0.94 \\
\hline
\end{tabular}

${ }^{*} P$-values are from comparisons between groups, using Wilcoxon or Chi-square as appropriate 
was typically a PI $(n=18)$ or NNRTI $(n=7)$, but two participants were taking an integrase inhibitor (raltegravir).

The percent changes in biomarker level by treatment group from baseline to month 1 and 6 follow-up time points are presented graphically in Figure 1. Compared to participants in the ABC group, the percent difference in biomarker levels for the TDF group at month 6 was $-79 \%$ for hsCRP (95\% CI $-205,-5 ; \mathrm{p}=0.05),-31 \%$ for IL-6 $(95 \%$ CI $-82,6 ; \mathrm{p}=0.12),-59 \%$ for D-dimer $(95 \%$ CI $-173,8 ; \mathrm{p}=0.11)$, and $-52 \%$ for the inflammation/coagulation rank composite (95\% CI -90 , -22; $\mathrm{p}=0.001)$. Corresponding treatment differences at month 1 visit were incomplete and not significant: $-41 \%$ for hsCRP $(\mathrm{p}=0.34), 4 \%$ for IL-6 ( $\mathrm{p}=0.92),-15 \%$ for D-dimer $(\mathrm{p}=0.41)$, and $-40 \%$ for inflammation/ coagulation rank composite $(\mathrm{p}=0.06)$.Treatment differences at month
6 were not attenuated after adjusting for 10 -year FRS: $-79 \%$ for hsCRP ( $\mathrm{p}=0.06),-35 \%$ for IL-6 ( $\mathrm{p}=0.11),-53 \%$ for D-dimer $(\mathrm{p}=0.15)$, and $-50 \%$ for inflammation/coagulation rank composite $(\mathrm{p}=0.004)$. Corresponding differences after adjusting for renal function (eGFR) were: $-79 \%$ for hsCRP ( $p=0.06),-35 \%$ for IL-6 $(p=0.11),-53 \%$ for D-dimer $(\mathrm{p}=0.15)$, and $-50 \%$ for the inflammation/coagulation rank composite $(\mathrm{p}=0.003)$. There was no treatment effect at 6 months on any of the biomarkers reflecting vascular inflammation (-4\% for sICAM-1 [95\% CI -23, 12], -6\% for ADMA [95\% CI -24, 9], 10.6\% for vWF [95\% CI $-9,25]$, and 5\% for sTM [95\% CI -16, 12]), or on the vascular rank composite $(1 \%, 95 \%$ CI $-16,12)$; comparisons also did not show significance at month 1 (data not shown).
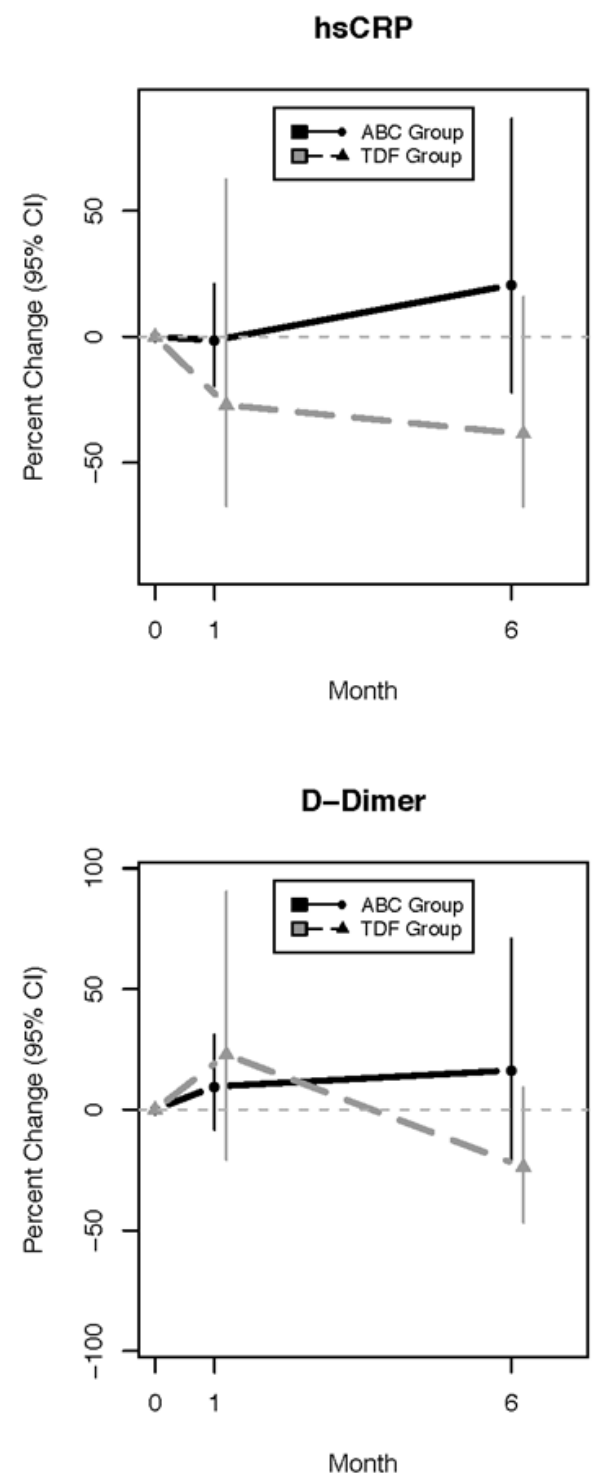

IL-6

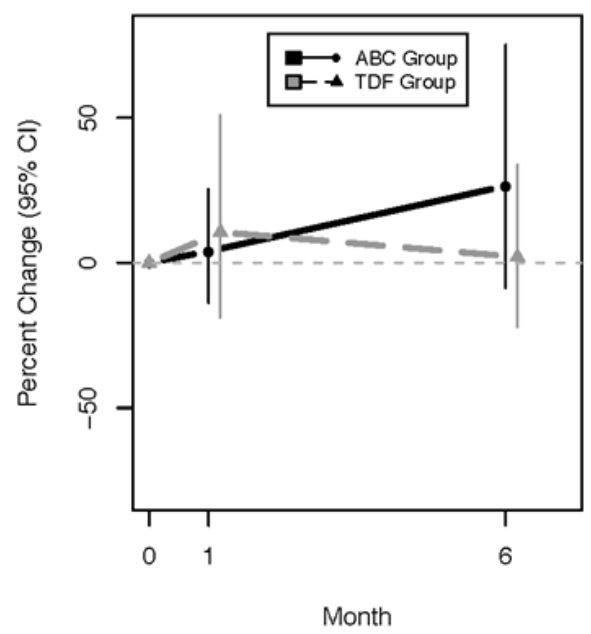

Inflammation/Coagulation Rank Composite

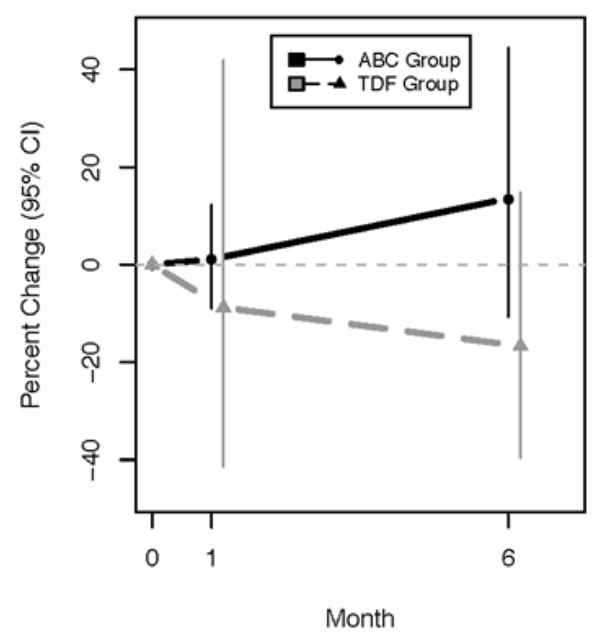

Percent changes from baseline are reported by treatment group for plasma levels of hsCRP, IL-6, and D-dimer and the inflammation/coagulation rank composite. Comparisons between treatment groups at each follow-up visit after adjustment for baseline level are reported in results

Figure 1: Percent Change in Biomarkers of Inflammation and Coagulation after Continuing Abacavir/Lamivudine (ABC Group) versus switching to Tenofovir DF/ Emtricitabine (TDF Group). 


\section{Discussion}

In this proof of concept study, we randomized HIV-positive participants with viral suppression taking fixed-dose abacavir/ lamivudine-based ART to remain on their current regimen or switch the nucleoside component to tenofovir DF/emtricitabine. Participants switching to tenofovir DF had significantly lower hsCRP levels and a lower inflammation/coagulation rank score after 6 months, when compared to those that stayed on abacavir. These pilot data raise the possibility that there may be greater systemic inflammation among patients on stable abacavir regimens. Though the clinical implications of these findings are unclear, the plasma biomarkers and rank score were chosen based on epidemiologic data demonstrating associations between these biomarkers and risk for CVD and all-cause mortality among HIV positive patients [13-16].

Our study provides additional randomized data among virally suppressed patients and are consistent with findings from the larger AIDS Clinical Trials Group (ACTG) study A5224s of starting ART [17]. In this study of 244 participants, hsCRP levels were higher 24 and 96 weeks after starting abacavir/lamivudine compared with those starting tenofovir DF/emtricitabine. A similar effect was reported in a nonrandomized comparison within the Strategies for Management of AntiRetroviral Therapy (SMART) study, where patients receiving abacavir had higher baseline hsCRP and IL6 (interleukin-6), compared with patients receiving other study NRTIs [18]. In another non-randomized comparison of 57 ART-treated participants with suppressed viral load, hsCRP levels increased 3 months after switching to an abacavir containing regimen but no increase was seen after 12 months or among those who switched to a tenofovir DF-based regimen [19].

Conversely, several other studies have failed to show any differential effect on inflammatory biomarker outcomes between abacavir and other NRTIs [20-23]. In the Multicenter AIDS Cohort Study (MACS) and Women's Interagency HIV Study (WIHS) cohort, none of the inflammatory biomarkers studied (hsCRP, IL-6 and $\mathrm{D}$-dimer) were higher among participants starting abacavir versus other NRTIs [20]. The BICOMBO study compared 80 ART-treated virally suppressed participants randomized to switch their NRTI backbone to either abacavir/lamivudine or tenofovir/emtricitabine, and found no difference in inflammatory (including hsCRP levels) and vascular inflammation biomarkers [21]. Null biomarker findings were also reported in the Simplification of antiretroviral therapy with Tenofovir-Emtricitabine or Abacavir-Lamivudine (STEAL) study, a randomized study of switching to abacavir- versus tenofovir DFbased ART regimens [22]. Importantly, the STEAL study also included a virally suppressed target population and the clinical endpoint analyses did find an association between abacavir use and CVD risk. This discrepancy in the STEAL study may be explained by alternative mechanisms for the potential abacavir-mediated CVD risk, other than inflammation, include inducing platelet hyper-reactivity [24,25]. Ultimately, disparate findings from multiple studies to date highlight the need for clinical endpoint studies (e.g., of CVD events) to determine if there is a differential effect of abacavir on CVD risk.

Our study was limited by its small size, exploratory nature, and use of plasma biomarker outcomes. The risk for both type 1 and type II statistical error mean findings should be interpreted with caution. The study design is also unable to control for any carry-over effect (e.g., abacavir-related abnormalities that persist after the drug is stopped). Despite these issues, our findings highlight a potential proinflammatory effect of abacavir exposure. Larger randomized studies are needed to verify whether switching from abacavir-based ART reduces inflammation and corresponding changes in clinical risk for CVD.

\section{Funding}

Gilead Pharmaceuticals, investigator initiated and sponsored project. Study investigators were not employed or contracted by Gilead to conduct this study, and had full control over conduct of the study, including study design, data analysis and the content of this manuscript.

\section{Acknowledgements}

We would also like to thank all study participants and our research staff for their consistent hard work: Bette Bordenave, Deb Wood, Edie Gunderson, Rache Givot, Miki Olson, and Jack Hall.

\section{References}

1. Mocroft A, Reiss P, Gasiorowski J, Ledergerber B, Kowalska J, et al. (2010) Serious fatal and nonfatal non-AIDS-defining illnesses in Europe. J Acquir Immune Defic Syndr 55: 262-270.

2. Worm SW, Sabin C, Weber R, Reiss P, El-Sadr W, et al. (2010) Risk of myocardial infarction in patients with HIV infection exposed to specific individual antiretroviral drugs from the 3 major drug classes: the data collection on adverse events of anti-HIV drugs (D:A:D) study. J Infect Dis 201: 318-330.

3. Sabin CA, Worm SW, Weber R, Reiss P, El-Sadr W, et al. (2008) Use of nucleoside reverse transcriptase inhibitors and risk of myocardial infarction in HIV-infected patients enrolled in the D:A:D study: a multi-cohort collaboration. Lancet 371: 1417-1426.

4. Choi Al, Vittinghoff E, Deeks SG, Weekley CC, LiY, et al. (2011) Cardiovascula risks associated with abacavir and tenofovir exposure in HIV-infected persons. AIDS 25: 1289-1298.

5. Durand M, Sheehy O, Baril JG, Lelorier J, Tremblay CL (2011) Association between HIV infection, antiretroviral therapy, and risk of acute myocardial infarction: a cohort and nested case-control study using Quebec's public health insurance database. J Acquir Immune Defic Syndr. 57: 245-253.

6. Obel N, Farkas DK, Kronborg G, Larsen CS, Pedersen G, et al. (2010) Abacavir and risk of myocardial infarction in HIV-infected patients on highly active antiretroviral therapy: a population-based nationwide cohort study. HIV Med 11: 130-136.

7. Brothers $\mathrm{CH}$, Hernandez JE, Cutrell AG, Curtis L, Ait-Khaled M, et al. (2009) Risk of myocardial infarction and abacavir therapy: no increased risk across 52 GlaxoSmithKline-sponsored clinical trials in adult subjects. J Acquir Immune Defic Syndr 51: 20-28.

8. Lang S, Mary-Krause M, Cotte L, Gilquin J, Partisani M, et al. (2010) Impact of individual antiretroviral drugs on the risk of myocardial infarction in human immunodeficiency virus-infected patients: a case-control study nested within the French Hospital Database on HIV ANRS cohort CO4. Arch Intern Med 170: 1228-1238.

9. Ribaudo HJ, Benson CA, Zheng Y, Koletar SL, Collier AC, et al. (2011) No risk of myocardial infarction associated with initial antiretroviral treatment containing abacavir: short and long-term results from ACTG A5001/ALLRT. Clin Infect Dis 52: 929-940.

10. Cruciani M, Zanichelli V, Serpelloni G, Bosco O, Malena M, et al. (2011) Abacavir use and cardiovascular disease events: a meta-analysis of published and unpublished data. AIDS 25: 1993-2004.

11. Campo R, DeJesus E, Bredeek UF, Henry K, Khanlou H, et al. (2013) SWIFT: Prospective 48 Week Study to Evaluate Efficacy and Safety of Switching to Emtricitibine/Tenofovir from Lamivudine/Abacavir in Virologically Suppressed HIV-1 Infected Patients on a Boosted Protease Inhibitor Containing Antiretroviral Regimen. Clin Infect Dis 56: 1637-1645

12. O'Brien PC (1984) Procedures for comparing samples with multiple endpoints. Biometrics 40: 1079-1087.

13. Kuller LH, Tracy R, Belloso W, De Wit S, Drummond F, et al. (2008) Inflammatory and coagulation biomarkers and mortality in patients with HIV infection. PLoS Med 5: e203.

14. Duprez DA, Neuhaus J, Kuller LH, Tracy R, Belloso W, et al. (2012) Inflammation, coagulation and cardiovascular disease in HIV-infected individuals. PLoS One 7: e44454. 
Citation: Alozie O, Prosser R, Huppler Hullsiek K, Duprez D, Rhame F, et al. (2014) Switching from Abacavir/Lamivudine to Tenofovir DF/Emtricitabine Reduces Biomarkers of Inflammation: A Randomized Proof of Concept Study. J AIDS Clin Res 5: 278. doi:10.4172/2155-6113.1000278

Page 5 of 5

15. Ford ES, Greenwald JH, Richterman AG, Rupert A, Dutcher L, et al. (2010) Traditional risk factors and D-dimer predict incident cardiovascular disease events in chronic HIV infection. AIDS 24: 1509-1517.

16. Triant VA, Meigs JB, Grinspoon SK (2009) Association of C-reactive protein and HIV infection with acute myocardial infarction. J Acquir Immune Defic Syndr 51: 268-273.

17. McComsey GA, Kitch D, Daar ES, Tierney C, Jahed NC, et al. (2012) Inflammation markers after randomization to abacavir/lamivudine or tenofovir/ emtricitabine with efavirenz or atazanavir/ritonavir. AIDS 26: 1371-1385

18. Strategies for Management of Anti-Retroviral Therapy/INSIGHT; DAD Study Groups (2008) Use of nucleoside reverse transcriptase inhibitors and risk of myocardial infarction in HIV-infected patients. AIDS 22: F17-24.

19. Kristoffersen US, Kofoed K, Kronborg G, Benfield T, Kjaer A, et al. (2009) Changes in biomarkers of cardiovascular risk after a switch to abacavir in HIV1 -infected individuals receiving combination antiretroviral therapy. HIV Med 10: 627-633.

20. Palella FJ Jr, Gange SJ, Benning L, Jacobson L, Kaplan RC, et al. (2010)
Inflammatory biomarkers and abacavir use in the Women's Interagency HIV Study and the Multicenter AIDS Cohort Study. AIDS 24: 1657-1665.

21. Martínez E, Larrousse M, Podzamczer D, Pérez I, Gutiérrez F, et al. (2010) Abacavir-based therapy does not affect biological mechanisms associated with cardiovascular dysfunction. AIDS 24: F1-9.

22. Martin A, Amin J, Cooper DA, Carr A, Kelleher AD, et al. (2010) Abacavir does not affect circulating levels of inflammatory or coagulopathic biomarkers in suppressed HIV: a randomized clinical trial. AIDS 24: 2657-2663.

23. Patel P, Bush T, Overton T, Baker J, Hammer J, et al. (2012) Effect of abacavir on acute changes in biomarkers associated with cardiovascular dysfunction. Antivir Ther 17: 755-761.

24. Satchell CS, O'Halloran JA, Cotter AG, Peace AJ, O'Connor EF, et al. (2011) Increased platelet reactivity in HIV-1-infected patients receiving abacavircontaining antiretroviral therapy. J Infect Dis 204: 1202-1210.

25. Baum PD, Sullam PM, Stoddart CA, McCune JM (2011) Abacavir increases platelet reactivity via competitive inhibition of soluble guanylyl cyclase. AIDS 25: 2243-2248. 